\title{
BERICHT ÜBER EINE REISE VON FürSTENSTEIN NACH HAMbURG UND Umgebung ÜBer Görlitz, Muskau, Branitz, Berlin vom 25TeN August bis 10ten SePtember 1869
}

\author{
Gärtner Kuhns, mit einem Vorwort von Justyna Jaworek-Jakubska
}

$\mathrm{D}$ en vorliegenden Bericht über eine Reise von Fürstenstein nach Hamburg und Umgebung über Görlitz, Muskau, Branitz, Berlin vom 25ten August bis 10ten September 1869 " $^{1}$ verfasste der Gärtner Kuhns aus dem fürstlichen Garten zu Fürstenstein bei Waldenburg (Wałbrzych) in Schlesien. ${ }^{2}$

Dem Reisebericht wird zunächst kurz die geschichtliche Entwicklung der fürstlichen Gartenanlagen in Fürstenstein vorangestellt, da diese den Wirkungsbereich Kuhns von 1855 bis 1892 bildeten und zudem versucht, seine Person näher zu beleuchten.

Anfang des 18. Jahrhunderts wurde die damals befestigte Ritterburg Fürstenstein zu einem repräsentativen Adelssitz umgewandelt. In der Regierungszeit von Konrad Ernst Maximilian von Hochberg (16821742) begannen die Arbeiten zur Anlage eines Ziergartens neben der Barockresidenz und die Umgestaltung des Popelberges, wo ein Lusthaus errichtet wurde. Die malerische Lage des Schlosses auf dem steilen Felsenvorsprung über der Schlucht des Hellebachs (Polsnitz) veranlasste Hans Heinrich VI. (1768-1833) zur Erweiterung der Gartenanlage. Auf seine Initative hin wurde auch der ausgedehnte romantische Landschaftspark im Polsnitzer Tal angelegt. In diesem Zeitraum entstanden zahlreiche romantische Bauten, darunter die Alte Burg, sowie Spazierwege und Aussichtspunkte, wie das »Riesen-Grab « (Abb. 1). ${ }^{3}$

Die großartigen Arbeiten an der Entstehung der Terrassengärten, die Kuhns nicht ohne Stolz in seinem Reisebericht mehrfach erwähnte, wurden von Hans Heinrich X.
(1806-1855) und von Hans Heinrich XI. (1833-1907) verfolgt. Hans Heinrich XV. (1861-1938) und seine Gattin Daisy von Pleß (Mary Theresa Olivia Cornwallis-West) gaben dem Gesamtkunstwerk schließlich den letzten Schliff. Sie begannen die umfangreiche Erweiterung des Schlosses. Die vorhandenen Gartenterrassen wurden modifiziert und weitere Terrassen neu angelegt (1905-1922). Damals enstanden die bis heute erhaltenen Gartenterrassen, wie Kaskadenterrasse, Mittelterrasse, Wasserterrasse (Abb. 2.), Kastanienterrasse, Hufeisenterrasse und Westterrasse (Abb. 3). Darüber hinaus erfolgte die Erweiterung des Landschaftsparks in Richtung Freiburg, die Einrichtung einer Pferdezucht und die Anlage der größten Schaugärtnerei Ostdeutschlands in Liebichau. ${ }^{4}$

In der zweiten Hälfte des 19. Jahrhunderts, als Gärtner Kuhns hier wirkte, befand sich das Herzogtum Fürstenstein im Besitz des Fürsten Hans Heinrich XI. In diesem Zeitraum wurden die Arbeiten an der Umgestaltung der Gartenterassen und Parkanlagen mit der Beteiligung Kuhns unter der Leitung des Gartendirektors Neide aus Charlottenburg durchgeführt. ${ }^{5}$

Die vorhandenen Informationen über Kuhns in den Akten der Familie von Hochberg sind sehr gering. Es ist nur bekannt, dass er in den Jahren 1858-1888 zuerst als Schloßgärtner ${ }^{6}$ und später zwischen 1888 und $1892^{7}$ als Garten-Inspektor in Fürstenstein tätig war. Er signierte Gartensachen betreffende Briefe sowie Zeichnungen zur Umgestaltung der Gartenanlage (zum Beispiel eines Springbrunnens im Jahre 1872). 


\section{Reisebericht des Gärtner KuHNS I 869}

$\mathrm{Ab} 1866$ beteiligte er sich an der Errichtung der neuen Gärtnerei, die an Stelle des ehemaligen Wirtschaftshofs errichtet wurde. Im selben Jahr erstellte ein Maurermeister (C. Bücker) die Pläne für das Gärtnerhaus und die Wirtschaftsgebäude, während Kuhns die Zeichnungen für das »Projekt zur Umänderung der Mistbeete in Treibhäuser « anfertigte. Das Sortiment der Gärtnerei umfasste im Jahre 1865 verschiedene Arten und Sorten von Zierpflanzen, darunter Rosen,
Pelargonien, Kamelien, Azaleen, Rhododendren, Chrysanthemen, Petunien, Begonien und Amaryllis. ${ }^{8}$

Im Jahre 1869 unternahm Garten-Inspektor Kuhns eine Reise zur Internationalen Gartenbau-Ausstellung in Hamburg (Abb. 4.), ${ }^{9}$ die ihn auch nach Görlitz, Muskau, Branitz und Berlin führte. Der von ihm verfasste Reisebericht wird nachfolgend wiedergegeben.

Justyna Jaworek-Jakubska

\section{ACTA DER FÜRSTLICH PLESSSCHEN GENERAL DIRECTION AUF DEM FÜRSTENSTEIN BETREFFEND GARTENSACHEN. 1858 - 1872.ULTO.VOL. I (FOL.283FF)}

[Fol. 284] Am 25ten August trat ich meine Reise nach Görlitz an. Erwähnenswerthe Gärtnereien in der Stadt sind erstens die des Herrn Dammann, in welcher ganz besonders die schönen Cycas (Sagopalmen) be- merkenswerth sind. Die Exemplare, über hundert an der Zahl, sind alle gesund und kräftig: Sie werden vorzugsweise der Wedel wegen cultivirt, welche bei Begräbnissen verwendet werden. Außerdem sind schöne

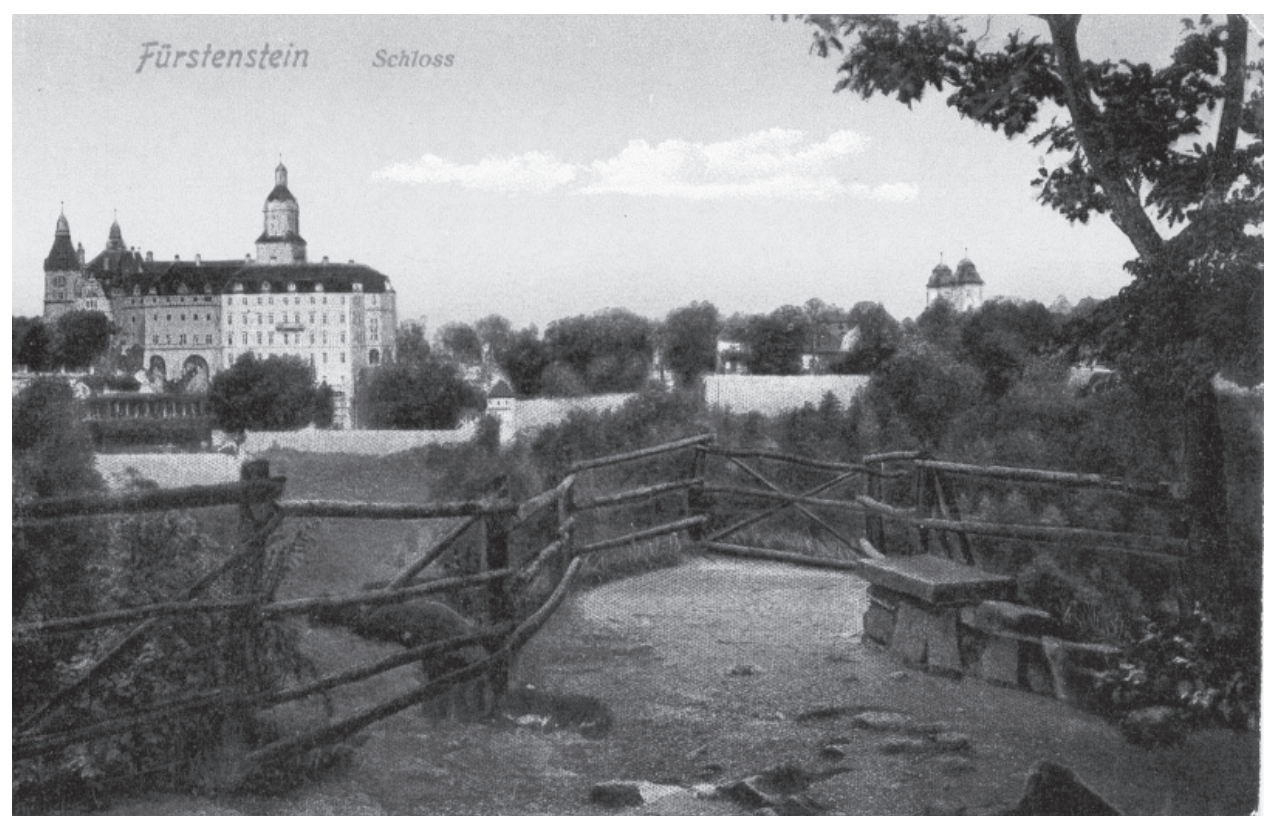

1 | Blick vom »Riesen-Grab« über das Hellebachtal zum Schloß Fürstenstein, Postkarte, Anfang des 20. Jh. (Stiftung der Fürstin Daisy von Pless). 
Ananas und Azaleen, auch ein Sortiment kräftiger Rosenstämme vorräthig. Von letzteren weniger neue Sorten.

Zweitens die Stadtgärtnerei mit geschmackvoll angelegten Promenaden unter Leitung des Herrn Promenaden-Inspectors Sperling. Die Anlagen, zu welchen auch der Berg Landskron bei der Stadt gehört, sind mit Verständnis angelegt, mit einigen Ausnahmen, welche aber durch die Verhältnisse zu entschuldigen sind. Sie werden sehr sauber gehalten und bieten sowohl an Laubwie an Nadelhölzern manches Gute dar. Auch ist die Teppichgärtnerei vertreten und in dieser, unter vielen anderen Pflanzen, eine Lawrencerose verwendet, welche mir noch neu war. Von letzterer Art habe ich ein Theil Stecklinge zur Anzucht für hiesige Teppichbeete erworben durch Tausch.

Görlitz ist überhaupt eine schöne Stadt und manche größere und eben so wohlhabende Stadt könnte sich in Betreff den Gartenanlagen an Görlitz ein Beispiel nehmen.

Am 27ten August reiste ich nach Bad Muskau. Die Stadt ist rings von dem weltbekannten Muskauer Park umgeben, letzterer ist eine Schöpfung Sr. Durchlaucht des Fürsten Pückler. Man sieht hier was sich durch Beharrlichkeit und natürlich die nöthigen Mittel schaffen läßt. Der jetzige Park war nämlich früher eine Sandfläche mit wenigen alten Bäumen als Bestand, welche meisterhaft bei der Anlage verwendet sind, es sind dieses meistens schöne Exemplare von Eichen; die anderen Gehölzgruppen und einzelnen Bäume sind in großen [285] Exemplaren gepflanzt. Man siehts dem Ganzen nicht an, daß es doch eigentlich noch eine neuere Anlage ist. Im Parke ist eine eigenthümliche Verwachsung, oder vielmehr eine Anwachsung einer Eiche um eine Buche. Man glaubt beim ersten Anblick, die Bäume seien miteinander verwachsen, sieht jedoch bei genauerer Untersuchung seinen Irrthum ein.

Eine noch neuere Abtheilung des Parkes ist das Arboretum. Hier sind die Gehölze erstens nach ihrem Vaterlande und zweitens nach ihrem botanischem System hainartig angepflanzt. Besonders auffällig war mir, daß hier die Wachsmyrthe, Myrica cerifera, auf ganz schlechtem Sandboden gedeiht,

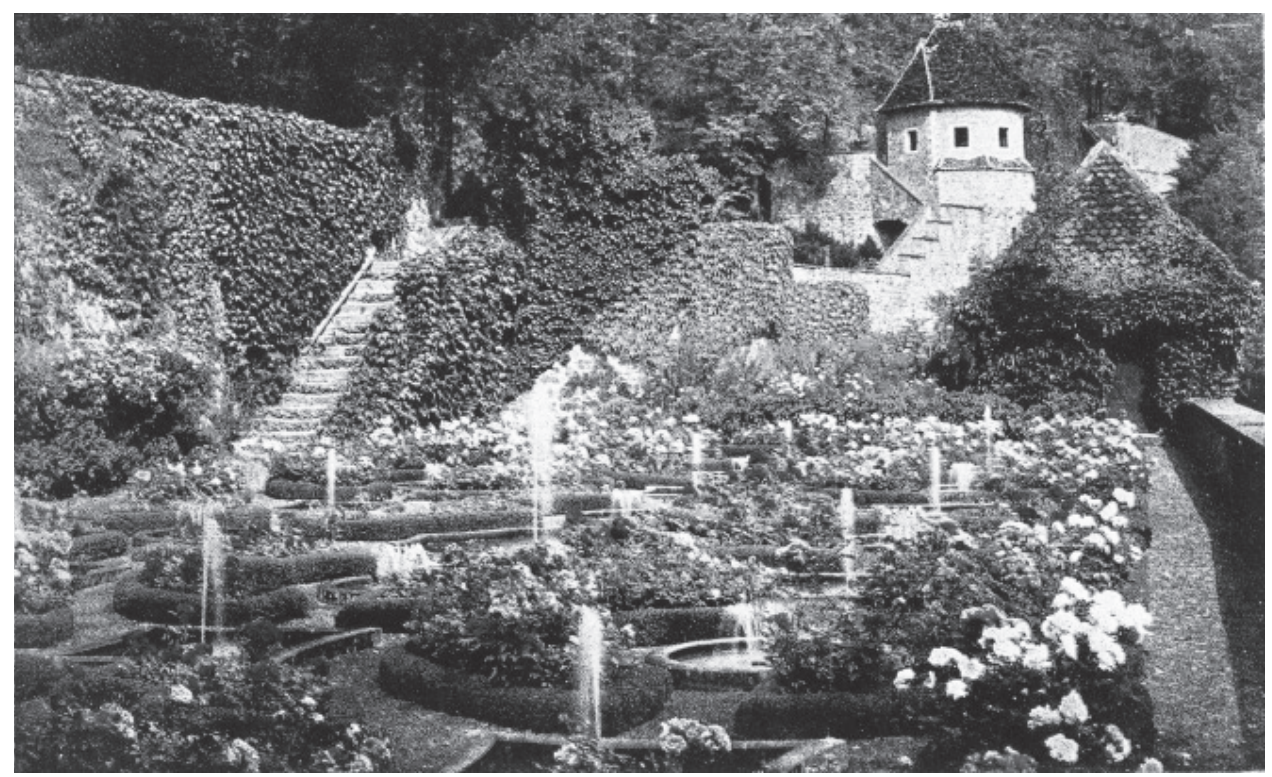

2 | Blick über die 27 Springbrunnen der Wasserterrasse, Postkarte, vor 1945, (Herder Institut in Marburg, Sign, 103262). 


\section{Reisebericht des Gärtner Kuhns I 869}

was man eigentlich von ihr nicht gewöhnt ist. Möglich, daß sich genannte Pflanzengattung auf solche Stellen anbauen ließe, wo nichts Anderes wächst, zum Zwecke der Wachsbereitung.

Vom Park eingeschlossen, hat Muskau auch eine sehr schöne Baumschule, sowohl Wild- als Stützbäume, von ersteren verdient besonders ein großes Eichensortiment der Erwähnung.

In der Gärtnerei sind die Topfpflanzen im guten Culturzustande auch in manchen neuen u. schönen Arten vertreten z. B. Ficus Cooperii, Dichorisandra mosaica, Maranta rosea picta, Caladium alba violacea, Cyanophyllum speciosum. Von genannten Pflan-

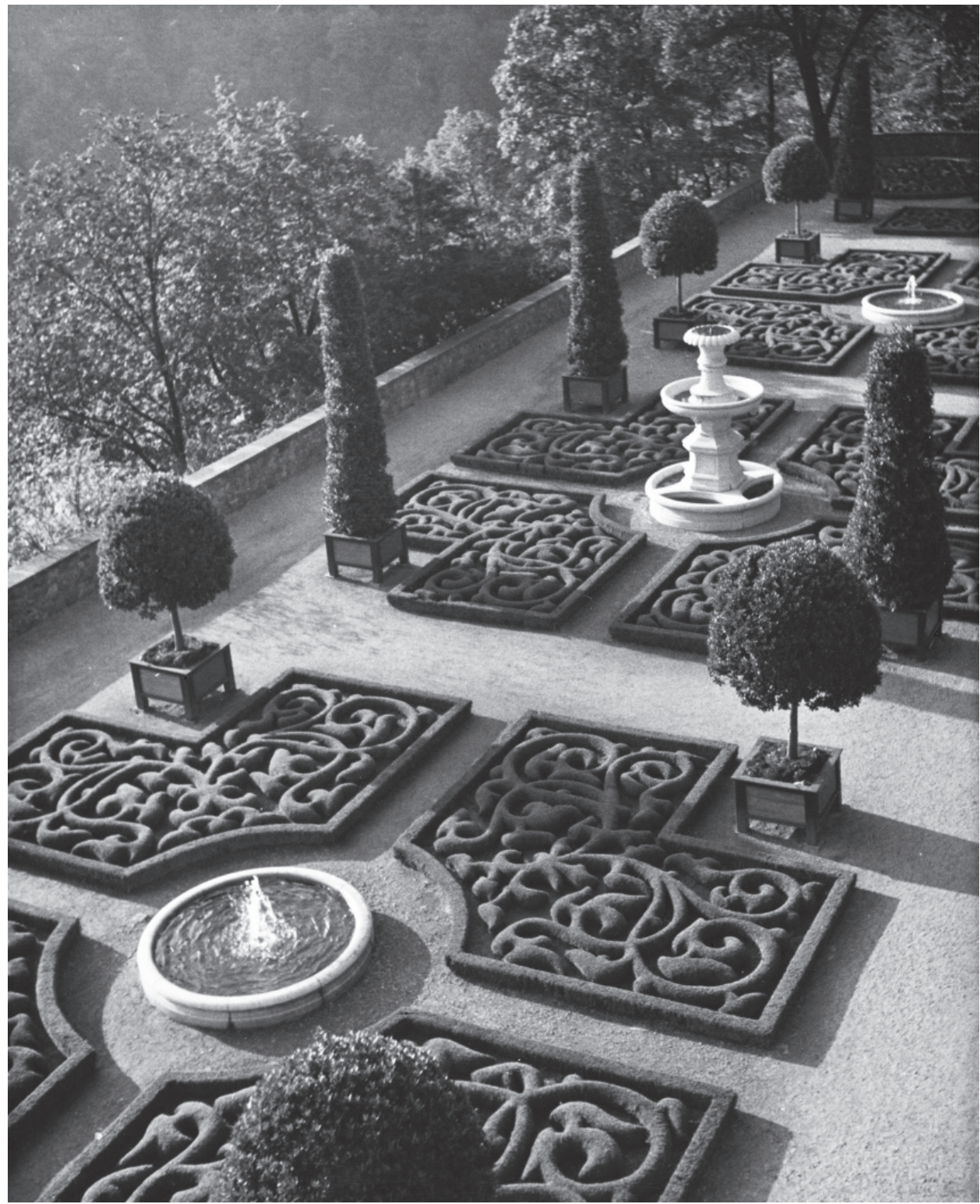

3 | Westterrasse, Fotografie, von Hitzig vor 1945, Quelle: Herder Institut in Marburg, Sign. 63918). 
zen sowie auch einige andere Arten, welche sich zur Teppichgärtnerei eignen, habe ich ein Theil im Werth von 15rthr. durch Tausch erworben.

Die nächste Umgebung des Schlosses war mit viel Blumengruppen und einzelnen Blumen- und Blattpflanzen geschmückt; ich möchte sagen: zu viel Blumen, es könnten [286] deren weniger sein ohne der Anlage zu schaden. Der Rasen ums Schloß war nicht besonders, ja man könnte sagen für die Teppichbeete schlecht.

Noch zu erwähnen ist das Bad, ein Theil des Parkes, es liegt mit der Vorderfront gegen die Stadt und legt sich mit der Hinterfront gegen den sogenannten Bergtheil des Parkes an. Er ist allerliebst angerlegt und obwohl ein für sich Abgeschlossenes doch mit dem Ganzen so verbunden, daß man es mit dem Ganzen zusammengehörig betrachten muß. Auch ist das sogenannte englische Haus im Parke mit seinen netten Umgebungen der Erwähnung werth. Hier stehen noch mehrere der noch ganz alten Eichen, welche schon vor der Anlegung des Parkes hier ihren Standort hatten.

Bei der Vorstellung des Parkinspector, wurde mir das Vergnügen zu Theil, mit dem Herrn Geheimrath Göppert aus Breslau bekannt zu werden. Er seinerseits freute sich auch, mich kennen zu lernen, sprach sein Bedauern aus, mich im Sommer nicht getroffen zu haben, als er, das ein Mal mit zwei Russen, u. das andere Mal mit Portugisen sich die neu geschaffenen Terrassen Fürstensteins ansehen wollte, aber abgewiesen wurde. Er beklagte es umso mehr, weil er gedachten Herren im vorhergehenden Frühjahr so viel von Fürstenstein erzählt hatte und sie aufgefordert hatte, sich die fast einzig dastehenden Terrassengärten anzusehen.

Mit der größten Bereitwilligkeit stellte uns der Herr Parkinspector Petzold seine Fuhre zur Disposition. Das Führeramt übernahm Vormittag von 7-9 Uhr der Obergärtner Kirchner, der genügenden Aufschluß über Alles gab, hinzu kam noch manche wissenschaftliche Erläuterung vom Herrn
Geheimrat Göppert, welches für mich sehr angenehm war. Seit langer Zeit habe ich dergleichen Unterhaltungen mit Fachmännern entbehren müssen.

[287] Nachmittag von 2-8 Uhr fuhr der Herr Parkinspector selbst mit. Wir sind fast den ganzen Tag im Parke herumgefahren $u$. nur an bemerkenswerthen Puncten abgestiegen. Um aber das Ganze genauer zu besehen, müßte man schon mehrere Tage dazu verwenden; denn in einem Tage ist's nicht möglich.

Den 28ten August früh fuhr ich in Gesellschaft der Herren Geheimrath Göppert u. des Herrn Parkinspector Petzold bis Kottbus. Die beiden erwähnten Herren fuhren nach Berlin u. ich machte von hier aus einen Abstecher nach Branitz, in die Anlagen des Fürsten Pückler [Abb. 5]. Der Park ist hier nichts so großartig wie in Muskau, auch noch nicht so herangewachsen, wenigstens nicht alle Theile desselben, aber dennoch ist er schön und wird in zehn Jahren in der üppigsten Laubpracht dastehen. Nach der Stadt Kottbus zu sind wieder neue Erdarbeiten in Angriff genommen um weitere Parkanlagen in Verbindung mit den alten zu schaffen. Leider ist der Herr Fürst Pückler in so vorgerücktem Alter, daß er wohl kaum das Ende dieser angefangenen Schöpfung erleben wird. Der nächste Theil des Parkes ums Schloss herum, ist reizend und gefällt mir viel besser als in Muskau. Es macht dieser Theil der Anlage einen so wohlthuenden u. befriedigenden Eindruck und man findet sich dort so heimisch, jedes Plätzchen nöthigt einem zum Niedersitzen, wenn man nur immer die gehörige Zeit hätte. Jeder Baum, jeder Strauch steht hier an seinem gehörigen Platze. Man ist überrascht durch die schönsten Bilder, welche durch die verständige Bepflanzung der Gehölze geschaffen sind. Das Ganze ist so schön harmonisch, man könnte sagen in einander verschlungen. Leider nimmt man sich, oder hat auch nicht immer Zeit, so etwas Gutes eingehender zu besichtigen.

[288] Am Abend des 28ten August fuhr ich nach Berlin. Neuerer Zeit ist hier die Baum- 
schule von Spät entstanden dann von Metz\&Comp: in Steglitz, erstere sehr sauber gehalten, dann eine der älteren großen Baumschulen von Lorberg, in dieser war ich im Jahre 1855 als Gehülfe beschäftigt. In letzterer Baumschule sind besonders schöne Nadelhölzer.

An Pflanzengärtnereien ist die schon längst bekannte des Herrn Borsig zu Moabit, dann von Reichenheim, Thiergarten. An Handelsgärtnereien, besonders für neuere Pflanzen, ist die des Herrn Benda, hier eine Sammlung guter Farne, Palmen u. neuere Blattpflanzen. Aus genannter Gärtnerei habe [ich] ein Theil gekauft u. für 25rthr. eingetauscht. Ein Hauptzweig sind hier die in neuerer Zeit so beliebt gewordenen Aquarien $\mathrm{u}$. Terrarien, von welchen auch von Herrn Benda in Hamburg ausgestellt waren und mit dem ersten Preis gekrönt wurden.

Allerliebst sind die kleinen Vorgärten in der Bellevue- $u$. Thiergartenstraße vor den oft prachtvollen Sommervillen. Sehr schön nimmt sich die Villa des Herrn Finanzministers v.d. Heydt am Canale aus, dieselbe ist mit einer reizenden kleinen Anlage umgeben, die auch sehr sauber gehalten wird.

Die Zeit vom 29ten bis 31ten August war für Berlin auch zu kurz. Manches hätte ich noch mitgenommen, doch es wurde zu viel. Am ersten September musste ich nun außerdem nach Fürstenstein zurück wegen Familien-Verhältnisse, wodurch mir zwei Tage verloren gingen.

Den 2ten September konnte ich nun ruhig u. ohne Besorgniß für meine Frau, nach Hamburg reisen. Dort angelangt war die erste Sorge um eine Droschke u. die zweite Sorge dann um eine Wohnung. Bei ersteren hatte man den Vortheil, daß wenigstens ein gewisser Taxpreis [289] feststand, bei letzteren dagegen war gar kein Harm [?]. Die Gasthäuser waren alle besetzt $u$. war nun auf Privatlogis angewiesen, von denen die Besitzer wirklich nicht wußten, wie hoch sie die Forderung stellen sollten. Nachdem ich endlich ein Lager gefunden, war der erste Gang in die Ausstellung.

Beim Eintritt in dieselbe wurde man von dem ersten großartigen Eindruck fast über-

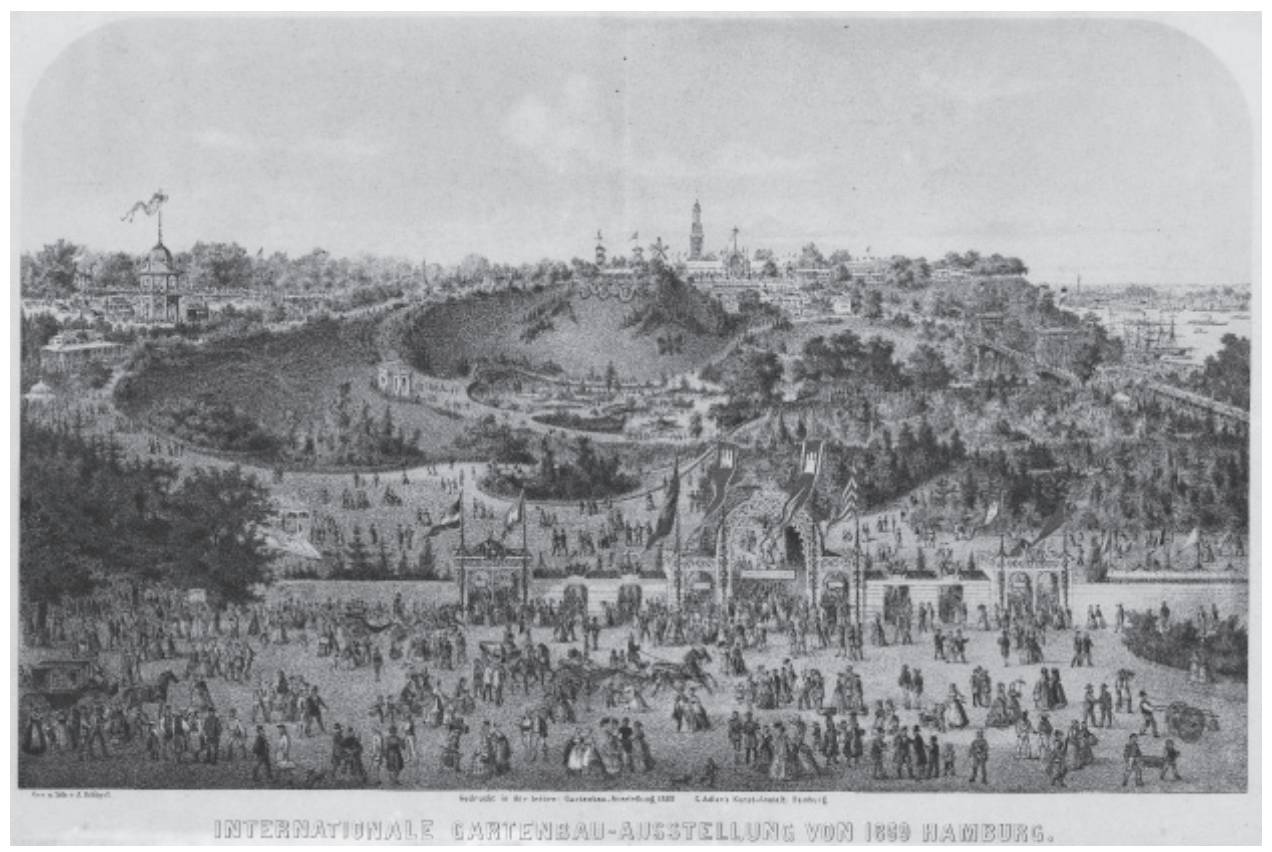

4 | Internationale Garten-Bau Ausstellung in Hamburg, 1869 (Staats- und Universitätsbibliothek Hamburg) 
wältigt. Man sah alles u. man sah Nichts. Bei specieller Besichtigung des Einzelnen fand sich doch auch manches Mangelhafte, letzteres war besonders der Fall auf der Hauptterrasse vor dem großen Warmhaus, dort war sowohl die Zeichnung als [auch] die Bepflanzung sehr mangelhaft. Ich kann mir eigentlich nicht erklären, weshalb man grade einen der Hauptpunkte so wenig Achtung geschenkt u. so wenig Fleiß darauf verwendet hat. Die Terrasse war in Form eines länglichen Vierecks, in der Mitte eine Fontaine, ein Fuß tiefer liegend als der Weg, angelegt. An beiden Enden war wieder ein Plateau, tiefer liegend als die ebengenannte Terrasse, auf diesen beiden Erhöhungen zwei runde Gruppen angebracht, von denen eine sehr hübsch war, die andere aber nur durch etwas gehoben wurde, daß man ein Rand weißen Sand streute. Dann war von Teppichbeeten in Form eines Dreiecks eines von Benda aus Berlin, sauber und geschmackvoll ausgeführt.

Zur besonderen Genugthuung u. Freude gereichten mir mehrseitige Äußerungen über die Fürstensteiner Terrassen am oben genannten Orte z. B. so was habe ich in Fürstenstein besser gesehen - das ist garnichts gegen Fürstenstein $\mathrm{u}$. dergl. mehr. Da ich doch von keinem der Umstehenden gekannt wurde bereitet mir das viel Spaß.

[290] An Pflanzenculturen waren besonders schön vertreten durch die Firmen: Verchaffelt, Linden, van Houtte aus Belgien; Wendland; Herrenhausen, Hannover; prachtvolle, gut cultivierte neuere Palmen, Orchideen, Droideau [?], eine Collection selten schöner Schlauchpflanzen, Baumfarne, zwei Stämme von gegen 20 [Fuß] hoch, eine Collection bunte Blattpflanzen von Benda-Berlin; gut culivierte Cycadeen [?]. Ein Sortiment von 150 Arten Cultur Farne u. fürs freie Land von Stelzner-Gent. Große Sortimente krautartiger Pflanzen: Fuchsien, Pelargonien, Heliotrop, Calceolarien [?] u.s.w. von ersteren aber kein so reichhaltiges Sortiment $u$. die Pflanzen nicht in solchem Culturzustande als in der hiesigen Gärtnerei.

An Gehölz u. Obstbäumen auch Conife- ren sind besonders hervorzuheben: Martin Müller-Straßburg; Spaet, Lorberg-Berlin; Ohlendorf-Hamburg; Jürgens-Nienstaedt, und von mehreren anderen, auch Muskau war vertreten. Vom Pomologischen Verein zu Booskop (Holland) Töllner-Schoenhof; Peter Smith\&Comp.-Hamburg; Born aus dem Othmarschen; Celloux\&fils, letzteres auch ein sehr gutes Obstsortiment, waren besonders schöne [...] ausgestellt. Ein Prachtexemplar von einer Araucaria impricata [?] von Verschaffelt.

Flor u. Modeblumen in allen Arten u. gut gezogenen Exemplaren von Verschiedenen aus allen Ländern besonders schöne Rosenstämme in kräftigen Exemplaren von Harms-Eimsbüttel, auch große gut gezogene Hochstämme zu Fuchsien - von demselben.

Abgeschnittene Blumen in allen möglichen schönen und häßlichen Formen gebunden, dergleichen in Sand getrocknete Blumen.

Eine große Sammlung verschiedener Sämereien [291] von Spreckelsen-Hamburg; dergleichen aus Guatemala.

Gepreßte Gemüse von Musson\&Comp. Hamburg; von Ganschnow-Dievitz; dann ein Sortiment getrocknete Producte von Krüger\&Sohn-Lübbenau; Rud. Lamm\& Comp.; Adler sen. Cöln.

Zwei Kartoffelsortimente, das eine von 75, das andere von 100 Sorten. Frisch Gemüse aller Art u. von letzteren ein besonders schönes u. gut cultivirtes Sortiment.

Ein Sortiment von 50 durchschnittener verschiedener Hölzer Australiens, ein Sortiment getrockneter Pflanzen eben daher, getrocknete Früchte, Algen u. Pilze von Goddeffroy Hamburg.

Eingemachte Früchte in Essig, Rum, Zucker u. Wein aus Bozen. Süd-Tyrol; desgleichen schöne Südfrüchte, Limonen u. Pumpelmuß von enormer Größe, Melonen allen [...].

Ananas von $53 \frac{3}{4} \mathrm{Pf}$. schwer, die größten welche ausgestellt waren. Zwar waren noch eben so schöne Früchte da, aber nicht so groß. Gut gezogene Obstbäume in Töpfen von Schwab-Darmstadt. Sehr große Wein- 
trauben aus England, Pfirsiche, Pflaumen, Aepfel, Birnen, sowie alle möglichen Obstsorten aus verschiedenen Gegenden.

Sehr bemerkenswerth waren des ObstKabinet von Arnoldi. Die Früchte sind aus Porzellancomposition täuschend ähnlich nachgemacht.

Nummerhölzer, ${ }^{10}$ Kisten, Schachteln, Stäbe, Körbe, Papiermanschetten in allen Formen und Größen.

Eine neue Art Nummerhölzer von Hartgummi, eine neue Composition, des gleichen Statuen von derselben Masse, sie sollen sowohl in Wasser als auch über der Erde gut halten, sowie harten Witterungswechsel trotzen.

[292] Eine auch noch neuere Art Nummerhölzer von Thon u. Steingut, in welchen die Namen eingebrannt werden, besonders zu empfehlen fürs Freie, aus Muskau, habe ich auch dort im Arboretum verwendet gefunden.

Zinkgußwaren von Kahle\&Sohn/Potsdam[,] Korb- u. Weidenmöbel von Henning Ahrens-Hamburg; von gehobeltem Eichenholz, sehr nett u. sauber gearbeitet u. zum Auseinandernehmen von André-Straßburg; auch geschmackvolle Möbel von Hirschgeweihen, vorzugsweise von Damschauflern, mit Hirschfellen überzogen von Rampendahl-Hamburg; aus gebogenem Holze von Thonet-Wien, von Eisen SeltenreichBerlin, -Mosenthin Petritsch [?], von letzteren war auch ein eisernes Glashaus in drei Abteilungen aufgestellt.

Eine große Auswahl schöner, geschmackvoller Pavillons, Laubengänge, Volieren, aus Holz, Eisen u. Draht. Vor allen waren ein Pa-

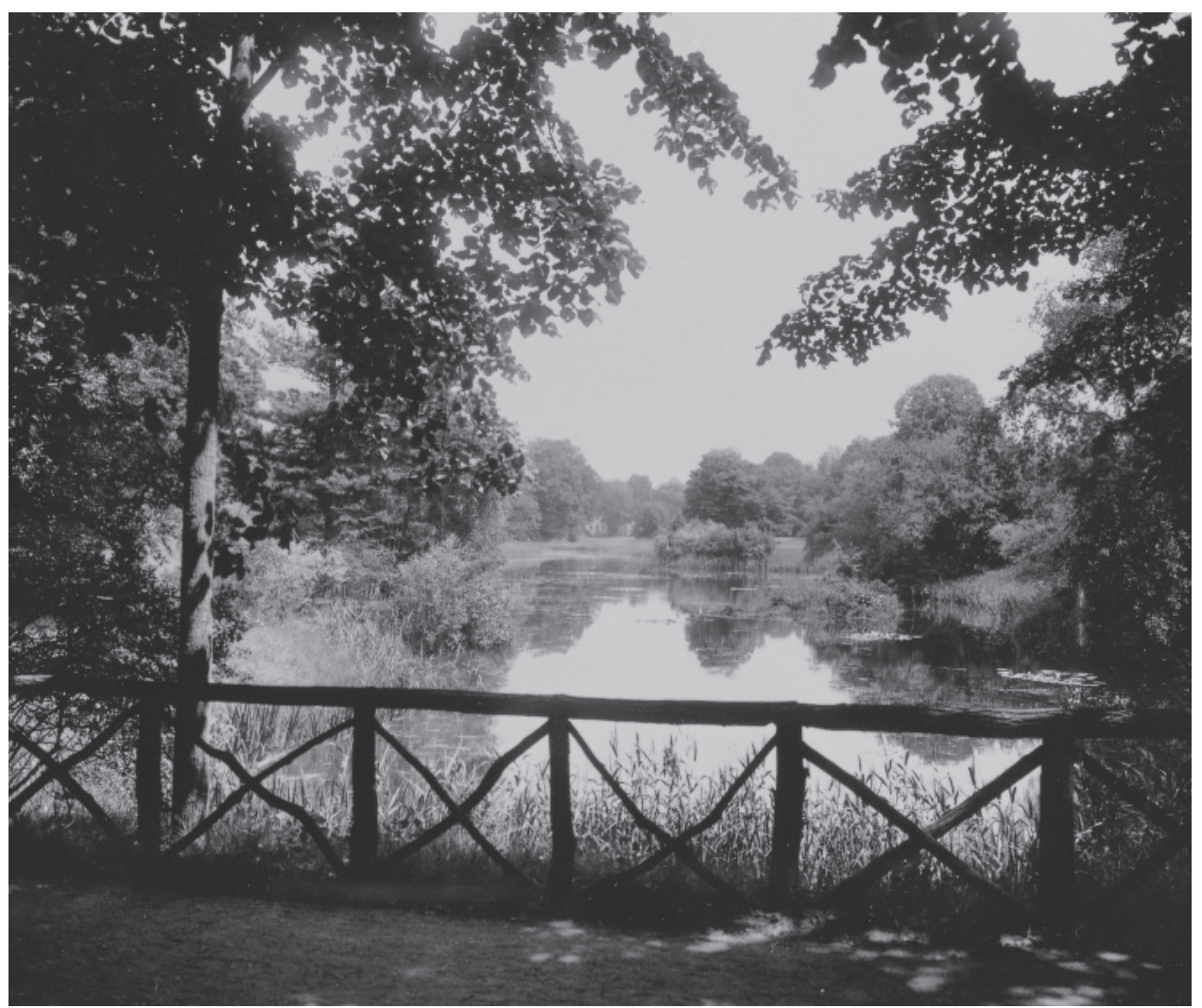

5 | Schlosspark Branitz, Fotografie, Regine Richter um 1910, (SLUB, Deutsche Fotothek, df_hauptkatalog_0281060). 
villon, reizend decorirt von Werner\&Pilgheim; von Holzdraht mit alten [?] Möbeln von Speyer Berlin.

Alabastervasen u. Töpfe, sowie auch prachtvolle Ampeln zur Beleuchtung, geschmackvoll in Form u. Farbe. Mosaikfußboden für Sommerhäuser u. Corridore in vielen schönen Mustern Dabelstein\&Comp.Hamburg.

Gruppen nützlicher $\mathrm{u}$. schädlicher Thiere für den Garten in Glaskästen von Dr. H. Landaus u. Dr. Altum [?] aus Münster. Ein Coniferen-Herbarium von HochstetterThüringen.

Brücken von Holz u. Eisen. Eine sehr dauerhafte Holzbrücke von sehr einfacher Construction, welche sich in sich selbst trägt. [293] Blumen von Blech, Gartenliteratur, Gartenpläne in der größten Auswahl.

Es ließen sich noch viele Gegenstände anführen, z. B. Gartenwagen, Garteninstrumente, Kannen, Spritzen, practisch u. unpractisch, und dergl. mehr. Blumentöpfe aus Kohle, Wasserhebewerke, Wasserheizungen und noch viele anderen Gegenstände. Es würde jedoch zu weit führen, alles einzeln aufzuführen.

Aus der Umgegend Hamburgs ist zunächst Uhlenhorst zu erwähnen. Es ist hier in die Anlage ähnlich der in der Thiergarten u. Bellevuestraße, nur daß dort die Häuser und Anlagen schöner sind wie hier. Die Häuser haben wie hier auch Land u. Sommerhäuser alle einen zu schweren Styl u. die Vorgärten sind nicht grad immer geschmackvoll angelegt noch gut gehalten. Einer der schönsten Besitzungen liegt grad über von Uhlenhorst über des äußern Alsterbassin. Das Haus ist dort im leichten hübschen Styl erbaut u. die Anlage vor dem Hause mit Verständniß ausgeführt sowie auch sauber unterhalten. Noch erwähnenswert sind noch die Privatbesitzungen von Fenisch [?] und Godeffroy.

Am 6ten September war ein großes Diner von sämtlichen Congreßmitgliedern in Blankenese. Im Ort selbst nichts Besonderes, nur daß das Terrain sehr hügelig ist und die Straßen sich, wie hier im Gebirge, in den
Thälern fortziehen. Schön ist die Aussicht vom höchsten Punct in Blankenese über die Elbe und besonders für mich Binnenländer ganz was Neues. Zwar bin ich schon im Jahre 1853 in Hamburg gewesen, traf aber so anhaltendes Regenwetter, daß von Ausgehen, um sich etwas anzusehen, garnicht zu denken war. Die Fahrt von Hamburg nach Blankenese per Dampfer war sehr angenehm und fürs Auge [294] wohltuend. Es liegen hier am Elbufer einzelne Besitzungen zwischen Laubholz versteckt, bald hoch, bald tiefer nach dem Wasser zu, bald nah, bald entfernter von einander ab, das Ganze ist landschaftlich schön. Abends $7 \mathrm{Uhr}$ wurde die Rückfahrt nach Hamburg angetreten. Das Comitée hatte das ganze Elbufer bengalisch erleuchten lassen, ebenso wurde jede Besitzung, sobald der Dampfer, auf welchem die Congreßmitglieder sich befanden, in die Nähe kam, was durch Signale angezeigt wurde mit bengalischen Flammen erleuchtet. Dazwischen Leuchtkugeln u. Raketen, vom Lande sowohl als auch vom Schiffe. Das Ganze machte einen imposanten Eindruck, beschreiben läßt sichs nicht.

Von Kiel ist in gärtnerischer Beziehung nichts zu erwähnen. Landschaftlich ist der ganze Hafen mit seinen ihn umgebenden Laubwaldungen. Von der Wilhelmmenhöhe-Bellevue genießt man eine weite Aussicht, von [?] weiter herauf von Friedrichsort. Je weiter man aber in die offene See gelangt, desto trister ist der Anblick. Die Ufer werden immer flacher, die Vegetation verliert sich mehr u. mehr, die Sandflächen werden immer größer, desgleichen die Wasserflächen und unwillkürlich beschleicht einen eine traurige Stimmung, noch zumal, wenns regnet und Wolken u. Wasser so ziemlich gleich aussehen. Eine Gebirgslandschaft ist doch schöner und bietet dem Auge mehr dar. Froh war ich als ich nach der Rückreise früh in Rabischau ankam, die Sonne ging eben auf und beleuchtete die ganze Gebirgskette, das war ein erquickender Anblick.

Dies war das Allgemeine von der Reise. 
[295] Durch die vielen Bekanntschaften von Fachmännern, war die Folge der tägliche Verkehr und der Austausch von Meinungen, sowie Mittheilungen von Erfahrungen in verschiedenen Zweigen der Gärtnerei, habe ich meine Kenntnisse um vieles bereichert. Auch hat man an manchen Orten kennengelernt, wie mans nicht machen soll.

Fürstenstein, den 26ten September 1869. Kuhns

(Transkription: Marcus Köhler)

Neide. Königlicher Gartendirektor in Berlin, Berlin 1884; Kurtz: Erinnerung an den am 28. August 1883 verstorbenen Königl. Garten-Direktor Eduard Neide, in: Garten Zeitung. Monatsschrift für Gärtner und Gartenfreunde, JG. 2 1883, S. 453, in: https://archive.org/stream/gartenzeitung02witt\# page/452/mode/2up (Stand: 26.09.1016).

6 General Direction auf dem Fürstenstein betreffend Gartensachen (1858-1872), Staatsarchiv Breslau Sygn. Hochb. II Gen. Dyr. 561, k. 391 und General der Fürstlichen Plessschen Central Verwaltung der freien Standesherrschaft Fürstenstein betreffend Gartensachen (1873-1883), Sygn. Hochb. II Gen. Dyr. 562, k. 350.

7 General der Fürstlichen Plessschen Central Verwaltung der freien Standesherrschaft Fürstenstein betreffend Gartensachen (1883-1888), Staatsarchiv Breslau, Sygn. Hochb. II Gen. Dyr. 563, k. 11-12, 98, 114, 120, 300, 306 und General der Fürstlichen Plessschen Central Verwaltung der freien Standesherrschaft Fürstenstein betreffend Gartensachen (1888-1892), Sygn. Hochb. II Gen. Dyr. 565, k.162, 246.

8 Staatsarchiv Breslau, Sygn. Hochb. II Gen. Dyr. 567, k. 210-238. Die Gärtnerei entwickelte sich in den 1890er Jahren stetig. Fördernd waren dabei vor allem die Kontakte mit anderen Gärtnereien in Breslau und Brieg (Staatsarchiv Breslau, Sygn. Hochb. II Gen. Dyr. 561, k. 169) sowie die Teilnahme an Obst- und Gartenbau-Ausstellungen in Breslau (Staatsarchiv Breslau, Sygn. Hochb. II Gen. Dyr. 565, k. 45-47).

9 Die Internationale Gartenbau-Ausstellung fand vom 2. bis 12. September statt (Illustrirter Führer durch die Internationale Gartenbau-Ausstellung zu Hamburg 1869, Hamburg 1869, S. 16). Dieser Führer, der auch eine Vielzahl an Illustrationen enthält, liegt digitalisiert vor: https://digital.slub-dresden. de/werkansicht/dlf/9312/6/.

10 »Unter Nummerholz versteht man ein kleines, ungefähr 1 Zoll breites, 4 bis 6 Zoll langes und mit einem Schnürchen versehendes Hölzchen, auf dessen glatt zugeschnittene Oberfläche man mit einem Bleistifte die Nummer und den Namen der Obstsorte schreibt.« (Seyfried, Johann Michael: Der pomologische Knabenfreund oder leichtfaßliche und gründliche Anleitung zur Obstbaumzucht, Innsbruck 1832, S. 41f.). 